\title{
Fetal Acalvaria Malformation: Antenatal Diagnosis in 4 Cases and Review of the Literature
}

\author{
Imane Attar ${ }^{1}$, Hekmat Chaara ${ }^{1}$, Hind Adadi $^{1}$, Sofia Jayi ${ }^{1}$, Fatima-Zahra Fdili Alaoui ${ }^{1}$, Moulay Abdelilah Melhouf ${ }^{1}$ \\ ${ }^{1}$ Department of Gynecology and Obstetrics Ii, Chu Hassan Ii Fes, Morocco
}

DOI: $10.36348 /$ sijog.2021.v04i04.005

| Received: 06.03.2021 | Accepted: 03.04.2021 | Published: 15.04.2021

*Corresponding author: Imane Attar

\section{Abstract}

Acalvaria Is a rare congenital disease considered as a post-neurulation defect: It consists of the absence of Calvary bones, dura mater and associated muscles in the presence of a normal skull base and facial bones normal. Currently, there is no identified cause of Acalvaria. The main putative pathogenesis is the problematic migration of the membranous neurocranium from the normal positioning of the immature ectoderm. Although the malformation has been fatal to date with only a few survivors, the prenatal diagnosis of Acalvaria is of rather remarkable importance as it allows clinicians to plan appropriate and timely management. So that this fetus can benefit from surgical advances.

Keywords: Acalvaria; antenatal ultrasound sign; differential diagnosis; prognosis.

Copyright () 2021 The Author(s): This is an open-access article distributed under the terms of the Creative Commons Attribution 4.0 International License (CC BY-NC 4.0) which permits unrestricted use, distribution, and reproduction in any medium for non-commercial use provided the original author and source are credited.

\section{INTRODUCTION}

Acalvaria is an extremely rare malformation characterized by the absence of the bones of the skull, dura mater and scalp muscles. The base of the skull and facial features are fully formed and generally appear normal [1]. It occurs at a frequency of 1 in 100,000 births [2]. Its causality is poorly understood and no concrete aetiology has been described [3].

This is an anomaly accessible to antenatal diagnosis from the 12th week of gestation in knowledge of the different differential diagnoses, of which anencephaly remains the most important [4]. This malformation can be associated with several abnormalities, which makes a detailed morphological examination mandatory in order to rule out any associated malformation which may further aggravate the postnatal prognosis [1].

Since then, Acalvaria has been widely regarded as a fatal anomaly [5]. But with advances in foetal medicine, prenatal identification of this anomaly has enabled clinicians to plan appropriate management resulting in a few surviving cases $[1,2,6,7]$.

Through four clinical observations and a review of the literature, we will prove the relevance of ultrasound in the prenatal diagnosis of this rare entity while raising the issue of differential diagnosis which presents itself as the main challenge for the obstetrician, as well as 'an overview on the prognosis will be reported.

\section{CLINICAL OBSERVATIONS Case $\mathrm{N}^{\circ} \mathbf{1}$}

This is a new born female, from a nonconsanguineous marriage, without ATCD (in particular no diabetes, no exposure to a teratogenic material), the pregnancy was not followed without any notion of premedication by acid folic, with a single ultrasound performed at 35 WA where the diagnosis of an anencephaly was made and then referred to our structure for specialist advice. Focused morphological exploration of the neural tube objectified a well-formed brain with cerebral convolutions, an interhemispheric fissure, lateral ventricles covered with a thick membrane that corresponds to the skin while the bone matrix was absent (Figure-1A). The facial structures appeared normal. The two orbital cavities were placed symmetrically of equal size and shape (Figure-1B). The brain showed a normal vascular pattern with normal function of Willis circle on the Doppler scan. No spinal defect was detected. The study of long bones seemed necessary to rule out osteogenesis imperfecta which 
Imane Attar et al; Sch Int J Obstet Gynec, Apr. 2021; 4(4): 103-107

was normal; and the length of the femur was consistent with the gestation period.

After informing the couples that it is a fatal malformation and given our religious context the Therapeutic Abortion was not discussed so a conservative attitude was adopted, with regular monitoring until the end to ensure fetal well-being, but essentially to detect the appearance of cerebral lysis which is only observed in Anencephaly, that will push us to question our initial diagnosis.

At 39 weeks the patient gave birth vaguely to a eutrophic mature newborn baby girl with normal hemodynamic constants, the morphological examination objectified a brain covered with a thick layer of skin, displaced towards the back due to the absence of supporting cranial bones including frontal, temporal, occipital and parietal bones, The facial structure and the rest of the body were completely normal. The evolution 2 hours later was towards death.

\section{Case $\mathbf{N}^{\circ} 2$}

Mrs HN, 36 years old, non-consanguineous marriage, first childbirth put on supplementation with folic acid from conception, referred in our training by her attending physician for suspected neural tube closure abnormalities and whose morphological ultrasound performed (25 week of amenorrhoea) objectified a complete protrusion of the early fetal brain above the remaining skull bones suggesting an Acalvaria malformation (Figure $1 \mathrm{C}, \mathrm{D}$ ), while the rest of the morphology was unremarkable. Regular monitoring was recommended until term with delivering through a cesarean section (for a surgical basin) giving birth to a newborn baby girl with Acalvaria who died 1 hour after.

\section{Case $\mathbf{N}^{\circ} 3$}

Mrs EL YF aged 29, not consanguineous marriage, followed for type I diabetes, first childbirth (folic acid administered from the 1st trimester), referred in our training for follow-up, whose morphological ultrasound in the 2nd trimester (26SA) objectified : the presence of a bone defect involving the entire skull with a complete protrusion of the fetal brain suggesting an isolated Acalvaria (Figure-1E). During monitoring, the patient presented an antepartum fetal death and delivered vaginally giving birth to a baby girl with Acalvaria.
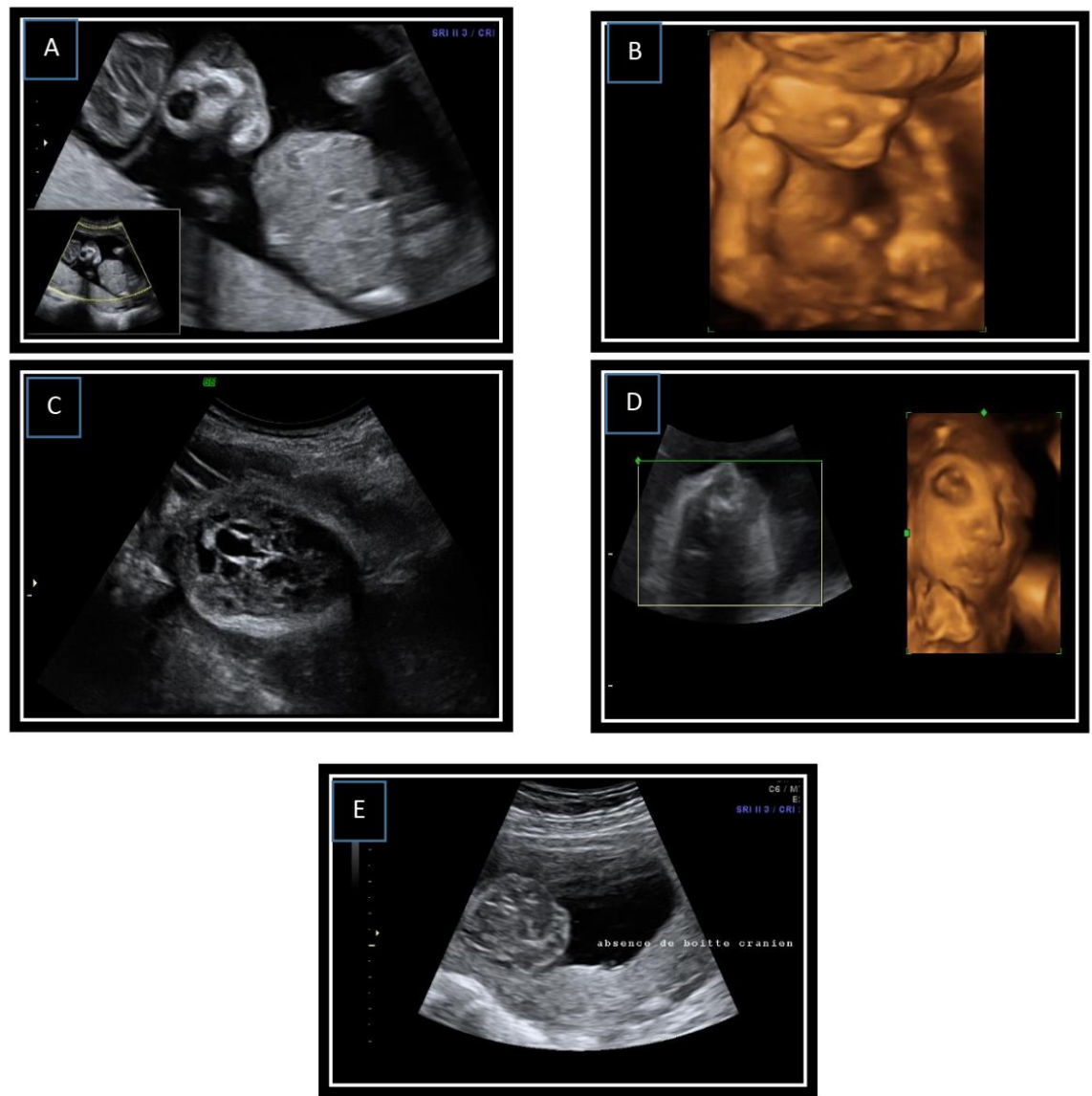

Fig-1: A-B) 2D and 3D ultrasound images illustrating agenesis of the cranial limb with preserved cerebral parenchyma in relation to Acalvaria in the first case.C-D) 2D and 3D ultrasound images illustrating agenesis of the cranial box with cerebral parenchyma preserved in report with an Acalvaria in the second case. E) $2 \mathrm{D}$ ultrasound image illustrating agenesis of the skull with preserved cerebral parenchyma in relation to an Acalvaria in third case 
Imane Attar et al; Sch Int J Obstet Gynec, Apr. 2021; 4(4): 103-107

Case $N^{\circ} 4$

Mrs AN, 36 years old, non-consanguineous marriage, with a history of two diabetic brothers on Oral Antidiabetics, first childbirth, referred in our training for management of a pregnancy associated with gestational diabetes discovered at 25 weeks by oral glucose tolerance test at 25 weeks +04 days. Whose morphological study this time has objectified a posterior segmental bone defect with protrusion of the cerebral parenchyma and a ventriculomegaly linked to the uterine wall by amniotic bands suggesting a partial Acalvaria secondary to an amniotic band (Figure-2), the patient reached full term gave birth vaginally to a new born baby girl whose Examination of the nervous system revealed partial agenesis of the occipital skull with adhesion between the cranial defect, the amnion and a layer skin covering brain tissue.
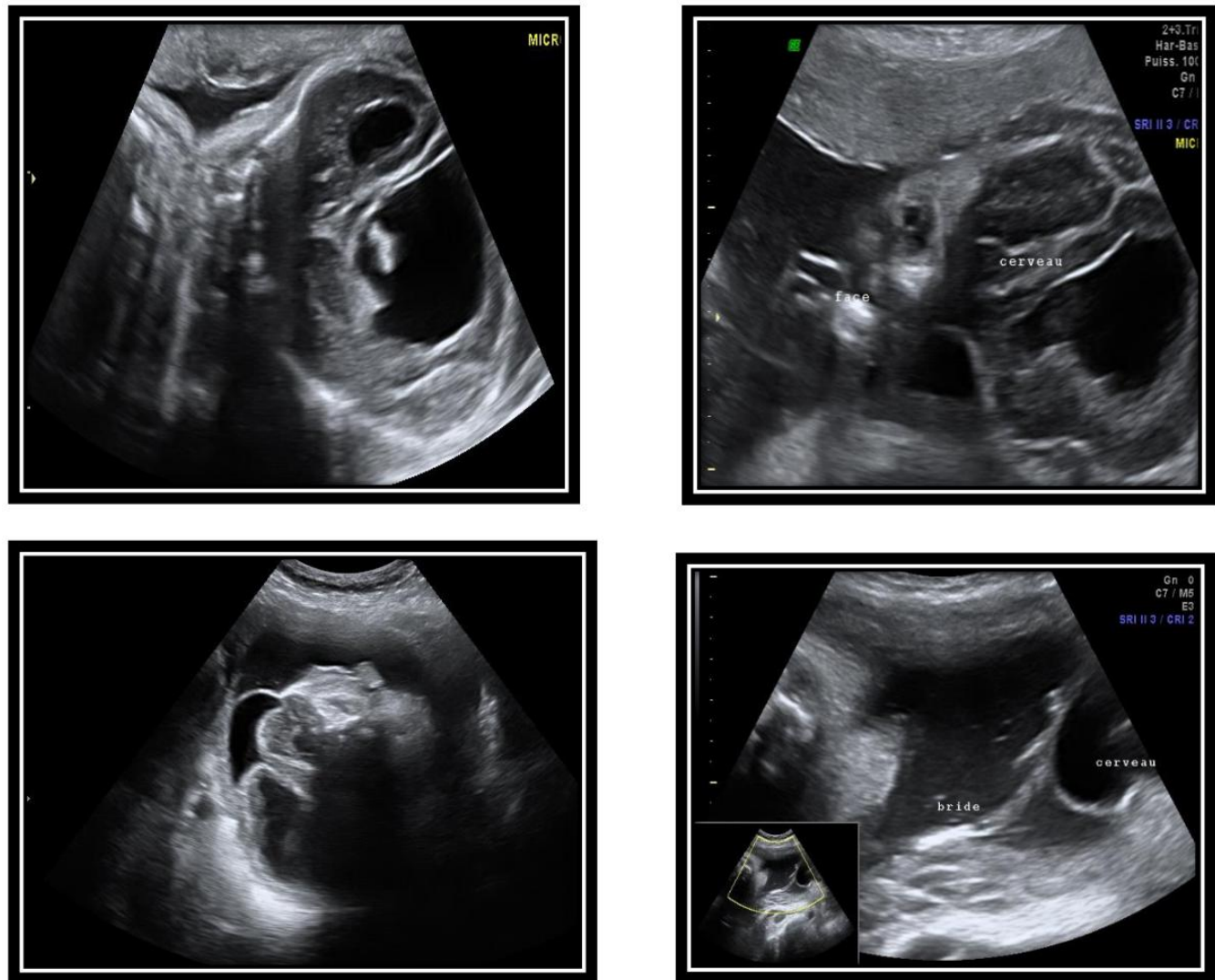

Fig-2: Ultrasound images showing partial agenesis of the cranial vault calling for Acalvaria, with adhesion between the cranial defect and the amnion covering the brain tissue

\section{DISCUSSION}

Lemire in 1988 divided neural tube defects into open (neurulation defects) and closed (postneurulation defects). Open lesions, thought to occur before neural tube closure in the embryonic stages, are much more common in foetal and pediatric neuropathology and include anencephaly and myelomeningocele. Post-neurulation neural tube defects are less common, including Acalvaria. The Acalvaria is characterized by the absence of flat bones of the skull, dura mater and associated muscles with the presence of normal cranial contents and facial bones. Although some cases may show abnormal development of brain tissue [8].

Acalvaria occurs in less than 1 in 100,000 births, only 23 possible cases have been reported in the English literature. Usually it is reported as a fatal congenital malformation with only rare cases (three in number) describing prolonged survival. The epidemiological investigation shows female predilection, as is the case in our series $[2,3]$.

This is a form of congenital malformation that result from poor migration of the membranous neurocranium (which gives rise to muscle and bone) with a normally placed embryonic ectoderm (from which the skin and scalp are formed). As a result, the brain is only covered with a layer of intact skin without a calvarium [9]. In cases associated with the amniotic band, the early rupture of the amnion is the triggering event. The amnion can twist like a cord trapping the head of the foetus [10]. Our fourth case illustrates this association.

So far, no etiology has been described. It has not been shown that taking folic acid prevents Acalvaria, which distinguishes this pathology from neural tube defects, confirmed by our study seen that 3 of our patients were on folic acid. Nevertheless, four of 
Imane Attar et al; Sch Int J Obstet Gynec, Apr. 2021; 4(4): 103-107

our patients were diabetic, this pathology which is often implicated in the abnormalities of the neural tube but no direct link with Acalvaria has been proven. There were no chromosomal abnormalities associated with Acalvaria, but other congenital anomalies can be associated with it such as holoprosencephaly, hydrocephalus as observed for our 4th case, micropolygyria, hypertelorism and cleft lip or cleft palate, for this the morphological analysis of the foetus must be thorough before predicting the prognosis $[1,3]$. Regarding our cases, no associated malformations were detected.

Detection of Acalvaria should be possible from the 12th week of gestation by trans-vaginal ultrasound. Early diagnosis is important for better management of the pregnancy. In our study, the mean age of diagnosis was 28 WA and no case was suspected in the first trimester due to the delay in consultation [1].

On ultrasound, the Acalvaria appears as an absence of posterior shading to the structures of the head due to the absence of the bony skull and dura mater and muscles with the presence of cranial contents covered by an echogenic layer that corresponds to the skin that protects the brain from degeneration with a normal facial floor, the whole achieves an aspect of "Mickey Mousse". Although some cases may show abnormal development of brain tissue, it is usually developed in the majority of cases. Anechoic areas representing necrosis or hemorrhage are also possible. This ultrasound description was the rule for making the diagnosis in our 4 patients.

A new means of antenatal diagnosis was suggested for Acalvaria is the dosage of alphafetoprotein (AFP) which should be normally low in these cases and whose rise is probably caused by the destruction of the tissue of the central nervous system by the amniotic fluid, something observed in the anencephaly, but this means of diagnosis remains a subject of debate since it can be distorted by the occurrence of AFP leakage through the thin layer of ectoderm covering the brain.

The differential diagnosis of Acalvaria remains a real challenge for the obstetrician. Acrania is the closest differential diagnosis and the two have been used interchangeably in the medical literature on several occasions. In Acalvaria, the brain being normal covered by the scalp and can potentially be treated with a chance of being compatible with life while the Acrania is lethal with brain tissue totally exposed to the outside. An incorrect initial diagnosis can be caught up with surveillance if the brain maintains its shape throughout pregnancy [2]
Other pathologies can be confused with Acalvaria such as [2]:

- Encephalocele: the cranial vault is still detected and part of the brain exteriorized.

- Severe osteogenesis imperfecta or Familial hypophosphatemia can lead to inadequate visualization or ossification of the bones of the calvarium, leading to a misdiagnosis of Acalvaria

- An additional condition that can be confused at autopsy with Acalvaria is aplasia cutis congenita (ACC). It is estimated that $20 \%$ of cases of ACC may have an underlying bony and dura defect thus causing possible diagnostic confusion.

The management of Acalvaria is not codified. The initial treatment is mainly conservative and aims at supportive care as well as the management of associated anomalies, no recommendation is declared in terms of surgical management. The treatment consists of an increase in soft tissues associated with a skin graft which will facilitate the protection of the dura by a layer of soft tissue while maintaining a separate layer of skin for future interventions, subsequently proceeded by a bone graft when the child reaches school age. There are only two cases published in the literature that have benefited from conservative treatment associated with extensive surgical reconstruction $[11,12]$

Usually, babies with this defect do not survive after birth. However, there have been cases of survival. The first surviving case was reported in 2004 in Japan. He was treated by surgical closure of the scalp defect, in addition, there was associated hydrocephalus, so shunt surgery was also performed. At follow-up, the child had severe developmental delay with mental retardation, last time reported alive at the age of 11 [3].

Unfortunately, in our series all our new-borns died after giving birth, which confirms the fatality of this anomaly.

\section{CONCLUSION}

Acalvaria is a rare congenital disease reported to be fatal in most newborns. It is an anomaly that remains accessible to antenatal diagnosis in knowledge of the different differential diagnosis that presents the main challenge for the obstetrician, her prognosis remains poor but there remains a malformation that deserves special attention so that it can benefit from progress. surgical nowadays.

Conflicts of interest: The authors declare no conflict of interest.

\section{Contributions from the authors}

All the authors contributed to the conduct of this work. All authors also declare that they have read and approved the final version of the manuscript. 


\section{REFERENCE}

1. Harris, C. P., Townsend, J. J., \& Carey, J. C. (1993). Acalvaria: a unique congenital anomaly. American journal of medical genetics, 46(6), 694-699.

2. Harris, C. P., Townsend, J. J., \& Carey, J. C. (1993). Acalvaria: a unique congenital anomaly. American journal of medical genetics, 46(6), 694-699.

3. Kurata, H., Tamaki, N., Sawa, H., Oi, S., Katayama, K., Mochizuki, M., ... \& Nakamura, H. (1996). Acrania: report of the first surviving case. Pediatric neurosurgery, 24(1), 52-54.

4. Ekici, E., \& Gülmezog̈lu, A. M. (1991). Sonographic diagnosis of fetal acrania. Journal of clinical ultrasound, 19(6), 363-366.

5. Bang, R. L., Ghoneim, I. E., Gang, R. K., \& Al Najjadah, I. (2003). Treatment dilemma: conservative versus surgery in cutis aplasia congenita. European journal of pediatric surgery, 13(02), 125-129.

6. Gupta, V., \& Kumar, S. (2012). Acalvaria: A rare congenital malformation. Journal of pediatric neurosciences, 7(3), 185.

7. Kamel, R. A., Ong, J. F., Eriksson, E., Junker, J. P., \& Caterson, E. J. (2013). Tissue engineering of skin. Journal of the American College of Surgeons, 217(3), 533-555.

8. Asai, M., Kitamura, H., Yanagibashi, T., Asukai, K., \& Katagiri, N. (1998). Case of acrania associated with congenital medulloblastoma. European Journal of Obstetrics \& Gynecology and Reproductive Biology, 81(1), 115-117.

9. Chandran, S., Lim, M. K., \& Yu, V. Y. H. (2000). Fetal acalvaria with amniotic band syndrome. Archives of Disease in Childhood-Fetal and Neonatal Edition, 82(1), F11-F13.

10. Sepulveda, W., De La Maza, F., \& Meagher, S. (2020). An Unusual First- Trimester Ultrasound Presentation of the Acrania- Anencephaly Sequence: The "Turkish Turban" Sign. Journal of Ultrasound in Medicine, 39(4), 829-832.

11. Hawasli, A. H., Beaumont, T. L., Vogel, T. W., Woo, A. S., \& Leonard, J. R. (2014). Acalvaria: case report. Journal of Neurosurgery: Pediatrics, 14(2), 200-202.

12. Bang, R. L., Ghoneim, I. E., Gang, R. K., \& Al Najjadah, I. (2003). Treatment dilemma: conservative versus surgery in cutis aplasia congenita. European journal of pediatric surgery, 13(02), 125-129. 